\title{
Calidad de vida y severidad de la adicción en heroinómanos en mantenimiento prolongado con metadona
}

\author{
Fernández Miranda, J.J.'; González Ga'-Portilla, M.P.'; Sálz Martínez, P.A.; \\ Gutiérrez Cienfuegos, E. '; Bobes García, J.2
}

(1) Unidad de Tratamiento de Toxicomanías. Servicios de Salud Mental de Asturias.

(2) Area de Psiquiatría. Departamento de Medicina. Universidad de Oviedo.

\section{Resumen:}

Se estudió la calidad de vida relacionada con la salud de 49 heroinómanos que llevaban 4 años de tratamiento en un PMM en Asturias, utilizando el SF-36. Además, la severidad de la adicción se midió con el EuropASI, y la sintomatología depresiva y/o ansiosa con la Escala de Ansiedad-Depresión de Goldberg (EADG).

Los pacientes mostraron una calidad de vida moderada, peor en las escalas del componente mental que en las del componente físicos (FF 86,5; RF 82,6; D 80,8; SG 52,4; V 46,4; FS 80.9; $\mathrm{RE} 68,4$; SM 58,6). Obtuvieron medias menores los $\mathrm{VIH}+$, los que habían consumido en los 6 meses previos heroína o elevadas cantidades de alcohol, y los que eran probables casos de ansiedad y/o depresión. La calidad de vida se correlaciona de forma inversa con la gravedad de la adicción. Se encontró mayor gravedad en las áreas de uso de drogas $(4,1 \mathrm{DE} 1,8)$, problemas psiquiátricos (3,2 DE 2,2), y situación médica (3,2 DE 2.4).

Además de mostrar peor calidad de vida, los VIH+ y los que mostraban comorbilidad psiquiátrica (ambos hechos se relacionaban) tenían mayor probabilidad de consumir drogas. Por tanto, se hace necesario incrementar y especificar las intervenciones psicosociales con este perfil de paciente para mejorar el cumplimiento del tratamiento con metadona en particular y su calidad de vida en general.

Palabras clave: Dependencia de heroína, mantenimiento con metadona, evaluación de tratamiento, severidad de la adicción, calidad de vida, comorbilidad psiquiátrica, VIH.

\section{Summary:}

We studied the quality of life level of a sample of 49 severe heroin-dependent patients involved in a methadone maintenance treatment in Asturias (Spain) over a 4 year period. Quality of life was assessed using the SF-36 (Spanish version). Severity of drugdependence was obtained employing the spanish version of the EuropASI, and symptoms of anxiety and depression were identified using the Goldberg's Anxiety and Depression Scale.

Patients showed moderate quality of life levels, with mental health scales worse than the physical ones (PF 86,5; RP 82,6; BP 80,8; GH 52,4; V 46,4; SF 80.9; RE 68,4; MH 58,6). Significantly lower levels of quality of life were found in HIV+ patients, in those who had consumed during the 6 months prior to the interview heroin or high doses of alcohol, and in those identified as probable cases of anxiety and/or depression. There was a significant inverse correlation between the severity of the addiction and the level of quality of life. The greater severity was found in the areas of drug use $(4,1$ SD 1,8), psychiatric status (3,2 SD 2,2), and medical status (3,2 SD 2.4).

Moreover, patients HIV+ and patients with psychiatric comorbidity (both facts were linked) were more likelihood to use drugs. It can be concluded that partients with this profile need more intensive and specific psychosocial support for a better global MMT compliance and higher quality of life level.

Key words: Heroin dependence, methadone maintenance, treatment evaluation, addiction severity, quality of life, psychiatric comorbidity, HIV.

\section{INTRODUCCION}

$\mathbf{L}$ os estudios sobre la efectividad de los programas de mantenimiento con metadona (PMM) se han centrado en el consumo de drogas ilegales, la situación somática y el aumento de la adaptación social de los pacientes, mostrándose exitosos en parámetros como disminución del consumo de drogas ilegales, disminución de la criminalidad, disminución de la morbi-mortalidad (especialmente la ligada al $\mathrm{VIH} / \mathrm{SIDA}$ ) y aumento de la actividad laboral, todo ello relacionado con una retención en tratamiento muy superior a otras modalidades $(1,2,3,4,5)$. 
Como vemos, las evaluaciones de PMM se han centrado en medir variables "duras" (consumo de drogas, morbi-mortalidad, criminalidad, empleo, etc.), pero no se han detenido a conocer el nivel de calidad de vida obtenido por los pacientes en el tratamiento, es decir, su percepción subjetiva. Aunque el término "calidad de vida" es cuestionado y de dificil precisión, existe un consenso sobre la concepción teórica de la salud como una valoración global del estatus individual, teniendo al bienestar como definidor de la misma, y la necesidad de calcular los efectos, tanto positivos como negativos, de las intervenciones sanitarias sobre las condiciones de vida de los enfermos y no sólo sobre los síntomas de la enfermedad (6).

Son ya numerosos los trabajos sobre calidad de vida en otras patologías, y varios los intentos de conseguir un instrumento de medida fiable. Destaca entre éstos el SF-36 Health Survey, adaptado para España no hace mucho (7). Parece ineludible, por tanto, medir un parámetro que se cita constantemente como objetivo final de los tratamientos con sustitutivos: la mejora de la calidad de vida del adicto $(8,9,10)$

La calidad de vida relacionada con la salud es un intento, pues, de evaluar el resultado funcional del impacto subjetivo de enfermedad y de su eventual tratamiento sobre un individuo concreto, que se convierte en su propio control comparativo. Aunque tiene una indudable relación con las discapacidades del sujeto y son parámetros que pueden interactuar, conviene recordar que son distintos (6). Por otro lado, los problemas derivados del consumo de drogas ilegales son variados y, muchos de ellos, con graves consecuencias para la salud, que conllevan importantes deterioros en la calidad de vida.

Como complemento a la medida de las variables objetivas, la percepción subjetiva del paciente, proporcionada por las escalas de calidad de vida, ofrece la posibilidad de plantear objetivos consensuados con él, además de una evaluación conjunta de los resultados. También ayuda a identificar déficits en áreas de funcionamiento y, por ende, la severidad de la adicción, facilitando así la identificación de grupos de riesgo y la planificación de medidas preventivas. En definitiva, junto con las variables "objetivas," ofrece una visión de conjunto de las necesidades del paciente, que es "escuchado", y de los resultados de las intervenciones sobre él.

En concordancia con lo expuesto, los objetivos de este estudio fueron: 1) Averiguar la situación de un grupo de heroinómanos a tratamiento en un PMM, tras cuatro años de continuar en el mismo (tanto la de consumo de drogas como la somática, psicológica, laboral y legal). 2) Medir la severidad de la adicción en ese momento. 3) Conocer la calidad de vida conseguida a los cuatro años de tratamiento. 4) Determinar las variables relacionadas con el cumplimiento del tratamiento por dicho grupo.

\section{PACIENTES Y METODO}

Se estudiaron los primeros 49 dependientes de opiáceos (criteros CIE-10) (11) que se mantuvieron durante 4 años en tratamiento en el PMM de la Unidad de Tratamiento de Toxicomanías del Area Sanitaria III de Asturias, abierta en 1991 y perteneciente a los Servicios de Salud Mental del Principado. El programa puede considerarse de medio-bajo umbral, enmarcándose los objetivos del tratamiento, sin descartar la abstinencia y el paso a un programa "libre de drogas", en la disminución de daños asociados al consumo. No existe lista de espera ni limitación temporal de tratamiento ni de dosis de metadona, siendo los criterios de inclusión los mínimos marcados por el real decreto de 1990 sobre tratamientos con sustitutivos opiáceos (12), y los de exclusión el consumo o tráfico de drogas y la violencia verbal o física en el centro.

Al comenzar el tratamiento se realizó una historia clínica completa, incluyendo variables sociodemográficas, clínico asistenciales previas e historial de consumo de drogas. Se estudió su estado físico con análisis de serología de VHB, VHC y VIH. Durante el seguimiento se realizó anualmente serología $\mathrm{VHB}, \mathrm{VHC}$ y $\mathrm{VIH}$ (utilizando EIA y Western-Blot) a todos los que continuaban en tratamiento.

Los consumos de heroína y cocaína fueron monitorizados durante el tratamiento, realizándoseles aleatoriamente a los pacientes tres controles de orina mensuales (mediante el método EMIT-DAU).

Las características sociodemográficas y clínicoasistenciales del grupo (al inicio y a los 4 años de tratamiento) quedan reflejadas en la tabla 1. El perfil del paciente es del de un varón, de 28 años de edad al empezar el tratamiento, heroinómano de larga evolución, consumidor de varias sustancias, que utiliza la vía intravenosa, con antecedentes penales, en paro, que convive con su familia de origen, con complicaciones médicas severas, y que ha realizado otros tratamientos de su dependencia con anterioridad al estudiado. La dosis media de metadona después de 4 años de tratamiento fue de 61,3 (DE 16,4 mg/día).

Al cuarto año de tratamiento los pacientes fueron evaluados con el Indice de Severidad de la Adicción (ASI), en su versión para Europea (EuropASI). Además, se les administró en esta entrevista de seguimiento la Escala de Ansiedad y Depresión de Goldberg (EADG), el cuestionario de alcoholismo CAGE y el de calidad de vida relacionada con la salud SF-36.

El hecho de administrar estos cuestionarios solamente en la entrevista de seguimiento a los 4 años, y 
Tabla 1.- Características demográficas y clínicas de los pacientes $(n=49)$

\begin{tabular}{|llll|}
\hline & Línea de Base & Año 4 & p \\
\hline Edad media & $28.6(4.8)$ & & \\
Sexo (varones) & $89.8 \%$ & & \\
Paro sin subsidio (\%) & $55.1 \%$ & $36.7 \%$ & \\
Conviven con familia de origen (\%) & $73.5 \%$ & $69.4 \%$ & \\
Conviven con alguien con problemas de drogas (\%) & $24.5 \%$ & $18.4 \%$ & \\
Juicios pendientes (\% si) & $32.6 \%$ & $10.2 \%$ & .007 \\
VIH + (\%) & $36.7 \%$ & $40.8 \%$ & \\
Consumo de heroína (\% si) & $100 \%$ & $36.7 \%$ & .000 \\
Vía intravenosa (\% si) & $93.9 \%$ & $24.5 \%$ & .000 \\
Consumo de cocaína (\% si) & $32.6 \%$ & $20.4 \%$ & .000 \\
Sobredosis (\% si) & $36.7 \%$ & $0 \%$ & .001 \\
Dosis de metadona (mg/d) & $49.4(18.5)$ & $61.3(16.4)$ & \\
\hline
\end{tabular}

no también al inicio del tratamiento, tiene una sencilla explicación: excepto el CAGE (que recordemos recoge problemas de alcohol a lo largo de la vida), el resto de los instrumentos o no existían en 1991-2 (EADG) o no estaban validados en España (SF-36, EuropASI). Aunque es evidente que el ideal pasa por poder comparar con los mismos instrumentos las dos situaciones, consideramos suficientemente interesante el haberlos administrado sólo en el seguimiento. Además, las variables estudiadas inicialmente son lo bastante variadas como para poder realizar una comparación en todas las áreas de los cambios "objetivos" del grupo estudiado, aunque no de su percepción de los mismos (medida con el SF-36).

EI ASI fue creado en EE.UU. en 1980, existiendo una versión para Europa desde 1995, adaptada de la $5^{\text {a }}$ edición americana por el grupo COST-A6 de la Unión Europea (13). Es una entrevista relativamente breve, semiestructurada y realizada para proporcionar información sobre aspectos de la vida del paciente que han podido contribuir a su sindrome de abuso de sustancias. Evalúa la severidad de la adicción en 7 áreas de la vida del paciente: situación médica, empleo/soportes, uso de alcohol, uso de drogas, situación legal, relaciones familiares/sociales, y estado psiquiátrico. A cada área se le asigna una puntuación de gravedad que oscila entre 0 (sin problema, sin necesidad de tratamiento) a 9 (problema extremo, tratamiento absolutamente necesario) (14).

Este instrumento ha sido utilizado ampliamente en Estados Unidos y ha demostrado ser un instrumento útil tanto en la práctica clínica como en investigación. Se ha constatado su validez concurrente y predictiva y su alta fiabilidad. Ofrece una serie de ventajas: relativa brevedad de aplicación, inclusión de todas las áreas comunmente afectadas en los adictos y posibilidad de utilización por diferentes profesionales entrenados. Se ha visto que las siete áreas son relativamente inde- pendientes entre sí. Quizás la información del área psiquiátrica sea la menos sensible, aunque esto pueda depender más que otras de la formación específica del entrevistador (14).

La Escala de Ansiedad y Depresión de Goldberg (EADG) es un instrumento de detección de probables casos de depresión y ansiedad. Sus ítems derivan de una conocida entrevista psiquiátrica, el Psychiatric Assesment Schedule, versión abreviada del Present State Examination (16). La versión española ha sido desarrollada por Lobo et al. a partir de la escala que el grupo de Goldberg diseñó en 1988. Como otros instrumentos de su género, no pretende diagnosticar más allá del nivel de "caso probable". La EADG ha demostrado ser un instrumento de "screening" muy válido para detectar con sencillez las patologías psiquiátricas más prevalentes entre los adictos: los cuadros depresivos y de ansiedad.

El cuestionario CAGE es una tradicional herramienta de detección de alcoholismo utilizada desde 1970. Este instrumento posibilita con comodidad, dado su contrastado poder diagnóstico (17), el despistaje de los problemas de alcohol entre los heroinómanos, especialmente relevantes en situaciones de abstinencia a la propia heroína.

Por último, el SF-36 fue desarrollado para su uso en el Estudio de Resultados Médicos en Estados Unidos (MOS) a partir de una extensa batería de cuestionarios que incluía 40 conceptos relacionados con la salud. Se seleccionó el mínimo número de conceptos para mantener la validez a la hora de crear el nuevo cuestionario. El SF-36 mide la calidad de vida relacionada con la salud mediante 36 ítems que se agrupan en las siguientes ocho escalas: Función física (FF), Rol físico (RF), Dolor (D), Salud general (SG), Vitalidad (V), Función social (FS), Rol emocional (RE) y Salud mental (SM) que se engloban en dos medidas sumarias de salud: el componente físico, constituido por las esca- 
las FF, RF, D y SG, y el componente mental constituido por las escalas V, FS, RE, y SM. Es necesario señalar que las escalas $S G$ y $V$ forman parte de ambos componentes, físico y mental. Cada una de las 8 escalas obtiene una puntuación que oscila entre 0 y 100 (del peor al mejor estado de salud) (15).

Su contenido, que incluye tanto la salud física como la mental, su robustez psicométrica y su relativa simplicidad y rapidez de administración lo convierten en un instrumento genérico de medida de calidad de vida relacionada con la salud muy útil en la evaluación de resultados clínicos (7). Además permite la comparación entre pacientes con la misma condición y entre diferentes condiciones.

Las definiciones de los dominios del SF-36 son:

-Función física: grado en el que la salud limita las actividades físicas.

-Rol físico: grado en el que la salud física interfiere con las actividades cotidianas.

-Dolor: intensidad del dolor y su influencia en la vida normal.

-Salud general: evaluación personal de la propia salud, incluso comparándola con la de los demás.

-Vitalidad: nivel de energía y vitalidad.

-Función social: grado en el que los problemas físicos o emocionales limitan las actividades sociales normales.

-Rol emocional: grado en el que los problemas emocionales interfieren en las actividades cotidianas.

-Salud mental: grado de síntomas depresivos o ansiosos y afectividad.

Todos los dominios del SF-36 fueron analizados respecto a variables demográficas, familiares, legales, laborales, médicas, psicológico-psiquiátricas, de consumo de drogas ilegales y alcohol, y de severidad de la adicción (ASI) a los cuatro años de tratamiento. Las pruebas estadísticas empleadas fueron: la ji-cuadrado con corrección de Yates (grados de libertad=1), la t de Student y el coeficiente de correlación de Pearson, según lo apropiado en función del tipo de variable (cualitativa o cuantitativa). El nivel de significación estadística quedó establecido en el 5\%. El tratamiento estadístico se realizó mediante el programa SPSS+.

\section{RESULTADOS}

Al inicio del tratamiento, el $100 \%$ de los pacientes consumía heroína, habiendo utilizado el $94 \%$ la vía endovenosa; el $54 \%$ abusaba de benzodiacepinas y el $32,6 \%$ de cocaína (tabla 1).

En la entrevista de seguimiento a los 4 años de iniciado el tratamiento, el $40 \%$ de los sujetos refirió no haber consumido nunca heroína en el año previo y el 76\% mantenerse abstinentes de cocaína en ese mismo periodo de tiempo. En los 6 meses previos a esta entrevista, los porcentajes de abstinencia fueron del $63,3 \%$ para la heroína y del $79,6 \%$ para la cocaína según los urinoanálisis, que muestran en ambos casos una clara tendencia hacia la abstinencia. Un $75,5 \%$ de los entrevistados refirió no haber utilizado nunca la vía intravenosa durante los últimos 6 meses para el consumo de drogas (opiáceos o estimulantes).

Los que habían consumido heroína en algún momento de los 6 meses previos al final del seguimiento eran mayores de 30 años $(p<0,05)$, tenían antecedentes legales $(p<0,05)$ y eran $\mathrm{VIH}+(p<0,01)$. Además, eran probables casos de ansiedad $(p<0.05)$ $y$, sobre todo, de depresión $(p<0,01)$ según la EADG.

Respecto al consumo de otras drogas ilegales durante el mes previo a la entrevista de seguimiento, el $72 \%$ refirió mantenerse abstinente de derivados cannábicos, el $92 \%$ de derivados anfetamínicos y el $66 \%$ de benzodiacepinas extraterapéuticas.

Por lo que se refiere al alcohol, el 81,6\% no bebía cantidades de riesgo (> $40 \mathrm{gr} / \mathrm{día}$ ), aunque sólo en el $49 \%$ resultó negativo el CAGE.

Ninguno de los sujetos había tenido reacción aguda a opiáceos ("sobredosis") durante el tratamiento. La seroconversión VIH se dió en 2 pacientes durante los 4 años estudiados, no constatándose ningún contagio de hepatitis $\mathrm{B}$ ○ $\mathrm{C}$.

La situación laboral (tabla 1) había mejorado, incrementándose ligeramente el nivel de empleo $(13,6 \%$ al $18,2 \%)$ y notablemente las jubilaciones $(17,4 \%$ al $34,1 \%)$. No obstante, en el $36,7 \%$ de los casos, el desempleo había sido su patrón habitual en los últimos tres años.

El 92\% refirieron no tener ninguna actividad ilegal en el mes previo. Un $89,8 \%$ no tenía causa judicial pendiente alguna ni estaba en libertad condicional. Ninguno de los entrevistados había estado en prisión en los últimos 6 meses. Estos datos reflejaron una mejora notable respecto a la situación inicial, en la que el 74\% tenía antecedentes penales, el 50 \% había estado en prisión y el $32,6 \%$ tenía causas pendientes (tabla 1).

Con el EuropASI se observó una severidad de la adicción muy moderada tras 4 años de tratamiento, siendo las áreas de uso de drogas $(4,1 ; D E 1,8)$, médica $(3,2 ; \mathrm{DE} 2,4)$, psiquiátrica $(3,2 ; \mathrm{DE} 2,2)$, y empleo/soportes $(2,9 ; D E 2,1)$ las más afectadas en comparación con la sociofamiliar $(2,6 ; D E 1,5)$, la de uso de alcohol $(1,9 ;$ DE 1,9) y la legal $(0,59 ;$ DE 1,2).

Los pacientes sin empleo presentaban significativamente mayor gravedad en las áreas de empleo/soportes $(p 0,000)$ y relaciones familiares y sociales $(p<0,01)$. Los pacientes $\mathrm{VIH}+$ presentaban mayor severidad en las áreas situación médica $(p$ 
$0,000)$, uso de drogas $(p<0,005)$, uso de alcohol $(p<0,05)$, situación legal $(p<0,05)$, relaciones familiares y sociales $(p<0,01)$, y psiquiátrica $(p 0,000)$. Del mismo modo, aquéllos que habían consumido heroína en los 6 meses previos a la entrevista presentaban mayor gravedad en las áreas de situación médica $(p<0,005)$, uso de drogas $(p<0,005)$, uso de alcohol $(p<0,01)$, situación legal $(p<0,01)$, relaciones familiares y sociales $(p<0,05)$, y psiquiátrica $(p<0,005)$. Con respecto al alcohol, los que consumieron $>40 \mathrm{~g} / \mathrm{d}$ durante los 6 meses previos a la entrevista obtuvieron puntuaciones más elevadas en las áreas uso de alcohol ( $p$ $0,000)$, relaciones familiares y sociales $(p<0,05), y$ psiquiátrica $(p<0,05)$, y los que fueron identificados como CAGE positivo presentaron mayor gravedad en las áreas de situación médica ( 0,000$)$, uso de drogas $(p<0,05)$, uso de alcohol ( $p, 000)$, relaciones familiares y sociales $(p<0,01)$, y psiquiátrica $(p<0,005)$. Los que fueron identificados como probables casos de ansiedad presentaban mayor gravedad en situación médica $(p<0,05)$, uso de drogas $(p<0,05)$, y problemas psiquiátricos $(p<0,005)$. Por su parte, los identificados como probables casos de depresión presentaban mayor severidad en las áreas de situación médica $(p<0,005)$, uso de drogas ( $p, 000)$, relaciones familiares y sociales $(p<0,005)$, y psiquiátrica. Finalmente, los que habían consumido heroína durante nueve años o más presentaban mayor severidad en el uso de alcohol $(p<0,05)$ y en las relaciones familiares $y$ sociales $(p<0,05)$ (tabla 2$)$.

Tabla 2. Severidad en el EuropASI [media (DE)] y diferencias significativas halladas..

\begin{tabular}{|c|c|c|c|c|c|c|c|}
\hline & $\begin{array}{l}\text { Situación médica } \\
3.2(2.4)\end{array}$ & $\begin{array}{c}\text { Empleo/soportes } \\
2.9(2.1)\end{array}$ & $\begin{array}{l}\text { Uso drogas } \\
4.1(1.8)\end{array}$ & $\begin{array}{l}\text { Uso alcohol } \\
1.9(1.9)\end{array}$ & $\begin{array}{c}\text { Situación legal } \\
0.5(1.2)\end{array}$ & $\begin{array}{c}\text { Familia/social } \\
2.6(1.5)\end{array}$ & $\begin{array}{l}\text { Psiquiátrica } \\
3.2(2.2)\end{array}$ \\
\hline $\begin{array}{l}\text { Situación laboral } \\
\text { (empleado vs desempleado) }\end{array}$ & n.s. & $\begin{array}{c}1.3(1.2) \text { vs } \\
4.9(0.9)\end{array}$ & n.s. & n.s. & n.s. & $\begin{array}{c}2.5(1.3) \text { vs } \\
3.5(1.1)\end{array}$ & n.s. \\
\hline $\begin{array}{l}\text { VIH status } \\
(\mathrm{VIH}-\text { vs VIH+) }\end{array}$ & $\begin{array}{l}1.5(1.2) \text { vs } \\
5.9(1.2)\end{array}$ & n.s. & $\begin{array}{l}3.5(1.8) \text { vs } \\
5.1(1.3)\end{array}$ & $\begin{array}{l}1.4(1.8) \mathrm{vs} \\
2.7(1.8)\end{array}$ & $\begin{array}{l}0.2(0.7) \text { vs } \\
1.2(1.6)\end{array}$ & $\begin{array}{c}2.2(1.7) \mathrm{vs} \\
3.3(1.1)\end{array}$ & $\begin{array}{l}\text { 2.3. (2.0) vs } \\
4.6(1.9)\end{array}$ \\
\hline $\begin{array}{l}\text { Uso heroína en los } 6 \text { meses } \\
\text { previos (no vs si) }\end{array}$ & $\begin{array}{l}2.3(2.2) \mathrm{vs} \\
4.5(2.2)\end{array}$ & n.s. & $\begin{array}{c}3.5(1.5) \text { vs } \\
5.0(1.6)\end{array}$ & $\begin{array}{c}1.4(1.7) \text { vs } \\
2.9(1.9)\end{array}$ & $\begin{array}{l}0(0) \text { vs } \\
1.2(1.5)\end{array}$ & $\begin{array}{c}2.2(1.3) \text { vs } \\
3.3(1.6)\end{array}$ & $\begin{array}{c}2.3(1.9) \text { vs } \\
4.5(2.1)\end{array}$ \\
\hline $\begin{array}{l}\text { Uso de } \mathrm{OH}>40 \mathrm{~g} \text { en los } 6 \\
\text { meses previos (no vs si) }\end{array}$ & n.s. & n.s. & n.s. & $\begin{array}{l}1.2(1.3) \mathrm{vs} \\
5.0(0.7)\end{array}$ & n.s. & $\begin{array}{l}2.4(1.5) \mathrm{vs} \\
3.6(1.3)\end{array}$ & $\begin{array}{l}2.9(2.2) \mathrm{vs} \\
4.7(1.9)\end{array}$ \\
\hline $\begin{array}{l}\text { CAGE screening } \\
\text { (negativo vs positivo) }\end{array}$ & $\begin{array}{l}1.9(2.0) \mathrm{vS} \\
4.5(2.1)\end{array}$ & n.s. & $\begin{array}{l}3.5(2.2) \mathrm{vs} \\
4.6(1.2)\end{array}$ & $\begin{array}{l}0.6(0.8) \text { vs } \\
3.1(1.8)\end{array}$ & n.s. & $\begin{array}{c}2.0(1.6) \mathrm{vs} \\
3.2(1.3)\end{array}$ & $\begin{array}{l}2.1(2.1) \mathrm{vs} \\
4.2(1.9)\end{array}$ \\
\hline $\begin{array}{l}\text { Ansiedad screening } \\
\text { (negativo vs positivo) }\end{array}$ & $\begin{array}{l}2.8(2.4) \text { vs } \\
4.7(2.0)\end{array}$ & n.s. & $\begin{array}{c}3.7(1.8) \text { vs } \\
5.2(1.1)\end{array}$ & n.s. & n.s. & n.s. & $\begin{array}{c}2.7(2.2) \mathrm{vs} \\
4.7(1.5)\end{array}$ \\
\hline $\begin{array}{l}\text { Depresión screening } \\
\text { (negativo vs positivo) }\end{array}$ & $\begin{array}{l}2.5(2.1) \mathrm{vs} \\
4.6(2.4)\end{array}$ & n.s. & $\begin{array}{c}3.5(1.7) \mathrm{vs} \\
5.3(1.2)\end{array}$ & n.s. & n.s. & $\begin{array}{l}2.2(1.5) \text { vs } \\
3.5(1.2)\end{array}$ & $\begin{array}{c}2.3(2.2) \mathrm{vs} \\
4.9(1.1)\end{array}$ \\
\hline $\begin{array}{l}\text { Duración del uso de heroína } \\
\text { ( }<9 \text { años vs } \geq 9 \text { años) }\end{array}$ & n.s. & n.s. & n.s. & $\begin{array}{l}\text { (1.2) vs } \\
2.7(2.1)\end{array}$ & n.s. & $\begin{array}{l}2.2(1.6) \text { vs } \\
3.1(1.4)\end{array}$ & n.s. \\
\hline
\end{tabular}

n.S.: no significativo

La EADG identificó un 24,5\% de probables casos de ansiedad y un $34,7 \%$ de probables casos de depresión. El hecho de ser detectado como probable caso de ansiedad se asociaba significativamente a consumo de heroína durante los 6 meses previos a la entrevista de seguimiento a los 4 años $(p<0,05)$, y a ser identificado como CAGE $+(p<0,05)$. Por su parte, ser clasificado como probable depresión se asociaba a ser $\mathrm{VIH}+(\mathrm{p}<0,01)$, y haber consumido $>40 \mathrm{~g} / \mathrm{d}$ de alcohol durante los 6 meses previos a la entrevista $(p<0,05)$.

La calidad de vida relacionada con la salud, medida con el SF-36, fue en general moderada, hallándose puntuaciones más bajas en las escalas del componente mental, vitalidad $(46,4 ; D E 13,8)$, función social (80,9; DE 21,0), rol emocional $(68,4$; DE 32,3), y salud mental $(58,6$; DE 15,4), que en las del componente físico, función física $(86,5 \mathrm{DE}$ 14,9), rol físico $(82,6 ; \mathrm{DE}$ $28,0)$, dolor $(80,8$; DE 19,9), y salud general $(52,4 ; D E$ 27,4).

En los pacientes $\mathrm{VIH}+$ la calidad de vida fue significativamente peor en las escalas de función física $(p<0,005)$, rol físico $(p 0,000)$, dolor $(p<0,005)$, salud general ( $p 0,000)$, vitalidad $(p<0,05)$, función social $(p<0,01)$, y rol emocional $(p<0,05)$. Los que habían 
consumido heroína durante los 6 meses previos a la entrevista obtuvieron peores puntuaciones en el rol físico $(p<0,05)$, salud general $(p<0,01)$ y salud mental $(p<0,05)$. Los que consumieron alcohol en cantidades de riesgo en los 6 meses previos a la entrevista tenían peores niveles de calidad de vida en rol físico $(p<0,01)$, dolor $(p<0,05)$, vitalidad $(p<0,01)$, función social $(p<0,05)$, rol emocional $(p<0,05)$, y salud mental $(p<0,01)$. Aquéllos que fueron identificados como probables casos de ansiedad en la EADG puntuaron inferior en dolor $(p<0,05)$, salud general $(p<0,05)$, función social $(p<0,01)$, rol emocional $(p<0,01)$, y salud mental $(p<0,005)$. Por su parte, los probables casos de depresión obtuvieron menor puntuación en función física $(p<0,005)$, rol físico $(p<0,005)$, dolor ( 0,000$)$, salud general $(p<0,005)$, vitalidad ( $p, 000)$, función social ( $p$ $0,000)$, rol emocional ( $p 0,000)$, y salud mental ( $p$
$0,000)$. Además, los que tomaban dosis de metadona mayores de $60 \mathrm{mg} / \mathrm{d}$ tenían peor nivel de calidad de vida en el rol emocional $(p<0,05)$. No se encontró relación significtiva entre la calidad de vida y el sexo, tipo de convivencia, patrón de empleo en los últimos tres años, o puntuación en el CAGE (tabla 3).

Cuando se correlacionaron las puntuaciones obtenidas en el EuropASI con las halladas en el SF-36, todas las áreas del EuropASI, excepto la de empleo/soportes y la situación legal, lo hacían de forma significativa e inversa. Los coeficientes de correlación más elevados se encontraron entre la situación médica del EuropASI y la escala de salud general del SF-36 (-.82). El coeficiente de correlación significativo más bajo correspondió al hallado entre el uso de alcohol del EuropASI y la escala de rol emocional del SF-36 (-.28) (tabla 4).

\begin{tabular}{|c|c|c|c|c|c|c|c|c|}
\hline & $\begin{array}{l}\text { FF } \\
86.5(14.9)\end{array}$ & $\begin{array}{l}\text { RF } \\
82.6(28.0)\end{array}$ & $\begin{array}{l}\text { D } \\
80.8(19.9)\end{array}$ & $\begin{array}{l}\text { SG } \\
52.4(27.4)\end{array}$ & $\begin{array}{l}V \\
46.4(13.8)\end{array}$ & $\begin{array}{l}\text { FS } \\
80.9(21.0)\end{array}$ & $\begin{array}{l}\text { RE } \\
68.4(32.3)\end{array}$ & $\begin{array}{l}\text { SM } \\
58.6(15.4)\end{array}$ \\
\hline $\begin{array}{l}\text { VIH status } \\
(\mathrm{VIH}-\mathrm{vs} \text { VIH+) }\end{array}$ & $\begin{array}{l}92.5(8.0) \text { vs } \\
77.7(18.0)\end{array}$ & $\begin{array}{l}95.6(16.4) \text { vs } \\
63.7(30.8) 0\end{array}$ & $\begin{array}{l}87.6(15.6) \text { vs } \\
71.0(21.7)\end{array}$ & $\begin{array}{l}68.4(17.2) \text { vs } \\
29.1(22.4)\end{array}$ & $\begin{array}{l}49.6(11.5) \text { vs } \\
41.7(15.8)\end{array}$ & $\begin{array}{l}88.2(16.4) \text { vs } \\
70.3(22.7)\end{array}$ & $\begin{array}{l}77.9(27.3) \text { vs } \\
54.7(34.6)\end{array}$ & n.s. \\
\hline $\begin{array}{l}\text { Uso de heroína en los } 6 \\
\text { meses previos (no vs si) }\end{array}$ & n.s. & $.94 .4(14.4)$ vs & $\begin{array}{l}\text { n.s. } \\
75.0(32.9)\end{array}$ & $\begin{array}{l}63.7(22.3) \text { vs } \\
39.0(29.0)\end{array}$ & n.s. & n.s. & $\begin{array}{l}\text { n.s. } \\
53.0(13.2)\end{array}$ & $63.8(15.0)$ vs \\
\hline $\begin{array}{l}\text { Uso de cocaína en los } 6 \\
\text { meses previos (no vs si) }\end{array}$ & n.s. & n.s & n.s. & n.s. & n.s. & n.s. & n.s. & n.s. \\
\hline $\begin{array}{l}\text { Cons. de } \mathrm{OH}>40 \mathrm{~g} \text { en los } \\
6 \text { meses previos (no vs si) }\end{array}$ & n.s. & $\begin{array}{l}88.1(24.4) \text { vs } \\
63.6(32.3)\end{array}$ & $\begin{array}{l}84.0(17.3) \text { vs } \\
69.7(24.8)\end{array}$ & n.s. & $\begin{array}{l}49.2(13.7) \text { vs } \\
36.8(9.8)\end{array}$ & $\begin{array}{l}85.0(18.9) \text { vs } \\
66.8(22.6)\end{array}$ & $\begin{array}{l}74.3(30.5) \text { vs } \\
48.0(30.9)\end{array}$ & $\begin{array}{l}61.6(14.9) \text { vs } \\
48.0(12.3)\end{array}$ \\
\hline $\begin{array}{l}\text { Ansiedad screening } \\
\text { (negativo vs positivo) }\end{array}$ & n.s. & n.s. & $\begin{array}{l}84.8(17.7) \text { vs } \\
68.5(21.9)\end{array}$ & $\begin{array}{l}57.2(25.5) \text { vs } \\
37.5(28.7)\end{array}$ & n.s. & $\begin{array}{l}84.3(18.7) \text { vs } \\
70.5(24.7)\end{array}$ & $\begin{array}{l}75.4(29.1) \text { vs } \\
46.9(33.1)\end{array}$ & $\begin{array}{l}61.7(15.5) \text { vs } \\
49.0(10.8)\end{array}$ \\
\hline $\begin{array}{l}\text { Depresión screening } \\
\text { (negativo vs positivo) }\end{array}$ & $\begin{array}{l}91.8(9.9) \text { vs } \\
78.1(17.6)\end{array}$ & $\begin{array}{l}95.0(13.7) \mathrm{vs} \\
63.1(33.7)\end{array}$ & $\begin{array}{l}88.9(15.9) \text { vs } \\
68.0(19.2)\end{array}$ & $\begin{array}{l}61.8(22.9) \text { vs } \\
37.5(27.9)\end{array}$ & $\begin{array}{l}52.3(13.0) \text { vs } \\
37.1(9.4) \\
\end{array}$ & $\begin{array}{l}92.8(10.8) \text { vs } \\
62.2(19.5)\end{array}$ & $\begin{array}{l}85.3(21.1) \text { vs } \\
41.7(28.9)\end{array}$ & $\begin{array}{l}66.9(12.7) \text { vs } \\
45.4(8.5)\end{array}$ \\
\hline $\begin{array}{l}\text { Dosis de metadona } \\
(\leq 60 \mathrm{~m} / \mathrm{d} \text { vs }>60 \mathrm{mg} / \mathrm{d})\end{array}$ & n.s. & n.s. & n.s. & n.s. & n.s. & n.s. & $\begin{array}{l}81.9(21.5) \text { vs } \\
62.5(34.6)\end{array}$ & n.s. \\
\hline
\end{tabular}

Tabla 4. Coeficientes de correlación de Pearson entre las áreas del EuropASI y las escalas del SF-36.

\begin{tabular}{|c|c|c|c|c|c|c|c|}
\hline & Situación médica & Empleo/soportes & Uso drogas & Uso alcohol & Situación legal & Familia/social & I Psiquiátrica \\
\hline Función física & -.66. & n.s. & -.44 & n.s. & n.s. & -.41 & -.55 \\
\hline Rol físico & -.67 & n.s. & -.48 & -.42 & n.s. & -.41 & -.63 \\
\hline Dolor & -.49 & n.s. & -.38 & n.s. & n.s. & -.36 & -.44 \\
\hline Salud general & -.82 & n.s. & -.45 & -.41 & n.s. & -.47 & -.70 \\
\hline Vitalidad & -.44 & n.s. & -.37 & -.44 & n.s. & -.36 & -.69 \\
\hline Función social & -.54 & n.s. & -.55 & n.s. & n.s. & -.53 & -.59 \\
\hline Rol emocional & -.36 & n.s. & -.43 & -.28 & n.s. & -.31 & -.53 \\
\hline Salud mental & -.38 & n.s. & -.46 & -.34 & n.s. & -.49 & -.68 \\
\hline
\end{tabular}




\section{DISCUSION}

Si ya son suficientemente reconocidas las dificultades para la evaluación de los tratamientos para la dependencia de sustancias, éstas se incrementan en los programas con sustitutivos opiáceos, pues la filosofía que los sustenta (9), los objetivos y el diseño de los mismos varían de un país a otro $(2,18)$, e incluso en el nuestro, de una comunidad autónoma a otra (19), de forma llamativa.

El perfil del paciente inicialmente atendido en el PMM es el de un heroinómano de larga evolución, con complicaciones médicas y escasa adaptación sociolaboral, y que ha realizado previos intentos de tratamiento. Este perfil coincide en general con el de los heroinómanos en PMM en España (20) y en Asturias (21). Cabe destacar, en lo que respecta a las diferencias de nuestro grupo con el "perfil español de pacientes de PMM", una menor prevalencia de infectados por el VIH, explicable por la menor tasa en Asturias de adictos $\mathrm{VIH}+$, seguramente en relación con el mantenimiento de los PMM durante los años 80; y un mayor nivel de desempleo, no sorprendente si consideramos la situación de declive industrial y elevado paro juvenil en el área por nosotros estudiada (Avilés).

Previamente a analizar los resultados de la variable "calidad de vida", creemos útil revisar las variables "duras".

Los resultados del EuropASI son congruentes con los hallazgos analíticos de consumo de drogas (urinoanálisis) y de estado físico (serología VIH). Y, en general, también con la apreciación subjetiva del paciente de su calidad de vida relacionada con la salud, medida con el SF-36.

Todos estos hallazgos nos muestran una situación muy mejorada respecto a la que el grupo estudiado ofrecía al comenzar el tratamiento, tanto en lo que respecta al consumo de drogas en general (drástica disminución del consumo de heroína, importante la del de cocaína, y cambios menos llamativos pero constatables hacia un menor uso de benzodiacepinas extraterapéuticas y cantidades elevadas de alcohol) como al nivel de reinserción social (importante disminución de problemas legales y más moderado incremento del empleo, muy condicionado por el contexto laboral del área). Además, las prácticas de riesgo asociadas al consumo son casi inexistentes, siendo la seroconversión VIH durante el tratamiento muy baja. Por último, los propios pacientes manifiestan tener una moderada satisfacción con su estado, atendiendo a los resultados del SF-36.

Estos cambios se pueden relacionar, razonablemente, con la permanencia en el PMM, aunque es evidente que una aseveración en este sentido pasaría por un estudio con "grupo control" sin tratamiento, a todas luces inviable por razones éticas. El hecho de que 49 pacientes se hayan mantenido en tratamiento durante 4 años seguidos demuestra, una vez más, la capacidad tan elevada de retención de los PMM (en concreto, la del estudiado es del $67 \%$ a los 4 años) y la necesidad de la misma para conseguir mejoras claras en la calidad de vida de sus pacientes.

Con respecto a los logros alcanzados durante el tratamiento con metadona, han sido varios los estudios sobre las variables que podrían incidir en la manifiesta efectividad de los PMM. Las más relacionadas con un peor pronóstico son el consumo de cocaína u otras drogas, la comorbilidad psiquiátrica, la dosificación de metadona inadecuada, el tiempo en tratamiento (retención) de menos de un año y la oferta asistencial escasa. $(2,4,10,18,22,23)$

En nuestra investigación se confirman algunos de estos aspectos, como son la efectividad general del programa y la influencia negativa que ejercen la comorbilidad psiquiátrica y somática $(\mathrm{VIH}+)$, y el abuso de otras drogas (alcohol) en la mejora de la situación de los adictos en el tratamiento, a pesar de un tiempo en el mismo suficiente ( 4 años) y una dosis de metadona adecuada (no se encontró relación alguna entre ésta y el resto de variables estudiadas).

Los resultados de nuestro estudio indican que los problemas de "salud mental" son aún importantes en poblaciones drogodependientes relativamente estabilizadas en tratamiento, ya que no sólo se reflejan en los dominios menos "físicos" del SF-36 y en el área de problemas psiquiátricos del EuropASI, sino que con la EADG se identifica un importante porcentaje de probables casos de ansiedad y de depresión, que concretarían las patologías psiquiátricas concomitantes.

Las investigaciones sobre calidad de vida en adictos fuera $(24,25,26)$ y dentro de nuestro país $(27,28)$, demuestran una mejora notable de la misma tras un tiempo en el PMM. Es llamativo el escaso número de investigaciones a este respecto, máxime cuando, como hemos comentado previamente, uno de los objetivos de los PMM es una "mejora general de la calidad de vida del adicto", que en la mayor parte de los casos parece medirse solamente con variables "duras".

Las dos experiencias más recientes que evalúan la calidad de vida en estos pacientes son la australiana, que muestra como los adictos al iniciar tratamiento con metadona tienen una calidad de vida mucho peor que la población general e incluso una salud física y mental tan pobre que es similar a la de grupos con enfermedades crónicas graves (somáticas y psiquiátricas) (26). Y la más cercana de Barcelona, que confirma una notable mejoría de la calidad de vida al año de estar en PMM, que ya se aprecia el primer mes, aunque sigue siendo peor que la de la población general de esa ciudad; y que la mejoría es menor especial- 
mente en aquellos consumidores de altas dosis de heroína (27).

Sin embargo, la medida de este parámetro es una incorporación relativamente reciente en los estudios de evaluación de resultados de tratamientos en general, salvo patologías como por ejemplo las tumorales, y quizás se deba esperar un tiempo a su utilización en el campo de las drogodependencias (6).

Aunque el instrumento para medirla sea diferente a los de los estudios sobre adictos ya comentados (salvo el de Ryan y White), los resultados obtenidos en nuestra muestra avalan la sensación, también por parte del adicto, de tener una aceptable situación, y que probablemente ésta tenga que ver con el hecho de permanecer en el PMM. Incluso ofrecen puntuaciones semejantes, y en algunos dominios mas altas, que las halladas en otros grupos a tratamiento por patologías crónicas, tanto psiquiátricas como somáticas en nuestro mismo medio $(29,30,31,32)$. No obstante, cabría decir que la calidad de vida de nuestro grupo es, sencillamente, diferente a la de otros, aunque ciertamente aún menor que la de la población general (7), probablemente debida en gran parte a los problemas somáticos (VIH) y psiquiátricos (depresión, ansiedad) concomitantes y a una dificultad notable de reinserción laboral.

En efecto, cuando se compara la calidad de vida de nuestros pacientes drogodependientes con la que presentan otros grupos de sujetos con patologías crónicas en Asturias, utilizando en todos los casos el SF36 , se aprecia que la de los pacientes drogodependientes es relativamente semejante a la de pacientes esquizofrénicos estabilizados en tratamiento; peor en las escalas del componente mental y mejor en las escalas del componente físico que la de los pacientes hemodializados; peor que los enfermos trasplantados de riñón en todas las escalas tanto del componente mental como del físico; y mejor en general (salvo en función social) que la de mujeres climatéricas sintomáticas. Estas comparaciones quedan reflejadas en la tabla 5.

Por último, realizando la comparación de nuestro grupo con la población española general de 25-34 años (7), vemos que sus puntuaciones son menores en todos los dominios, siendo las diferencias más acusadas en salud general, vitalidad, rol emocional, y salud mental (tabla 5); es decir, en las escalas más relacionadas con el componente mental de la calidad de vida relacionada con la salud.

Es necesario hacer hincapié en que la calidad de vida de nuestro grupo está muy condicionada, como era de esperar, por la infección por el VIH, y también por la presencia de psicopatología (ansiedad, y especialmente depresión), que la merman notablemente. Las relativamente bajas puntuaciones en salud general, vitalidad, rol emocional y salud mental están probablemente relacionadas con estos hechos.

Por otra parte, hay que destacar que la información aportada por el SF-36, es decir, la valoración subjetiva del paciente, no es exactamente "paralela" a la reflejada por los datos objetivos (análisis, situación laboral y legal) o por los instrumentos de evaluación valorados por el clínico, y en cierto modo considerados como "objetivos" (EuropASI). Así, aunque los datos "objetivos" señalan la importante influencia en los resultados del tratamiento de los problemas con el alcohol, del hecho de estar infectado por el VIH y de la ansiedad, los datos "subjetivos" (SF-36) apuntan a que lo más importante para el paciente, en cuanto a su influencia, son los trastornos depresivos y su condición de $\mathrm{VIH}+$. Es decir, el estudio de la calidad de vida puede ayudarnos a matizar y a priorizar los problemas a resolver según el interesado.

\section{Tabla 5. Comparación del nivel de calidad de vida [media (DE)] entre distintos tipos de enfermos crónicos y la población general española de 25 a 34 años de edad.}

\begin{tabular}{|c|c|c|c|c|c|c|}
\hline & $\begin{array}{l}\text { Drogodependientes } \\
\text { (F-Miranda et al) } \\
n=49\end{array}$ & $\begin{array}{c}\text { Esquizofrénicos } \\
\text { (Bobes et al) } \\
n=60\end{array}$ & $\begin{array}{l}\text { Trasplantados riñón } \\
\text { (Rebollo et al) } \\
n=186\end{array}$ & $\begin{array}{l}\text { Hemodializados } \\
\text { (Rebollo et al) } \\
\quad n=50\end{array}$ & $\begin{array}{c}\text { Climaterio } \\
\text { (del Fresno et al) } \\
n=69\end{array}$ & $\begin{array}{l}\text { Población general (25-34) } \\
\text { (Alonso et al) } \\
n=\imath \text { ? }\end{array}$ \\
\hline Función física & $86,5(14,9)$ & $89,1(15,7) *$ & $84,3(11,8)$ & $61,3(24,8) * *$ & $75,5(23,9)$ & $97,4(10,0) * *$ \\
\hline Rol físico & $82,6(28,0)$ & $63,7(39,9) *$ & $88,1(28,3)$ & $72,0(41,8)$ & $52,8(44,5) * *$ & $93,8(22,3)$ * \\
\hline Dolor & $80,8(19,9)$ & $80,0(24,4)$ & $80,3(24,7)$ & $65,6(26,5)$ & $57,8(24,4) * *$ & $89,4(19,8)$ * \\
\hline Salud general & $52,4(27,4)$ & $58,4(26,8)$ & $64,7(21,6) * *$ & $37,9(22,3) *$ & $49,0(25,5)$ & $78,6(16,6) * *$ \\
\hline Vitalidad & $46,4(13,8)$ & $53,4(24,6)$ & $74,4(17,5) *$ & $62,9(18,8) * *$ & $43,4(26,9)$ & $74,9(17,5) * *$ \\
\hline Función social & $80,9(21,0)$ & $70,0(29,9)$ & $93,4(14,5) * *$ & $60,3(27,3) *$ & $84,2(30,2)$ & $96,1(11,4) * *$ \\
\hline Rol emocional & $68,4(32,3)$ & $55,5(44,1)$ & $86,7(31,4) * *$ & $92,6(25,5) * *$ & $62,4(43,8)$ & $95,4(18,4) * *$ \\
\hline Salud mental & $58,6(15,4)$ & $60,0(24,2)$ & $79,0(16,6) * *$ & $75,2(15,0) * *$ & $51,3(23,3)$ & $77,9(16,8) * *$ \\
\hline
\end{tabular}




\section{CONCLUSIONES}

Los resultados globales obtenidos en el PMM evaluado pueden considerarse muy positivos, ya que han alcanzado con suficiencia gran parte de los objetivos adjudicados a este tipo de programas: disminución del consumo de drogas ilegales, prevención del deterioro físico y de los problemas legales y, en general, aumento de la integración social.

Desde el punto de vista del propio paciente se demuestra una aceptable calificación de su situación, con una calidad de vida moderada según el SF-36.

En cuanto a las variables relacionadas con la evolución, cabe destacar la necesidad de detectar y tratar lo antes posible la psicopatología asociada (depresión y ansiedad), pues condicionan una peor evolución con policonsumos (de drogas ilegales y alcohol) y menor calidad de vida. La relación de la psicopatología con la infección por $\mathrm{VIH}$ es probable en muchos casos, por lo que el incremento de apoyo psicosocial a estos pacientes se hace necesario.

La mejora en la eficacia del programa pasaría, por tanto, por una mayor atención a los problemas de salud mental de los pacientes, además del abordaje de la propia dependencia de heroína y de otras sustancias.

Por último, se constata que la medida de la calidad de vida es un "outcome" muy válido en la evaluación del tratamiento, así como su importancia en la matización de los resultados obtenidos por otros medios respecto a variables objetivas.

\section{BIBLIOGRAFIA}

(1) Simpson D, Sells S. Effectiveness of treatment for drug abuse: an overview of the darp research program. Alcohol Substance Abuse 1982, 2: 7-29.

(2) Arif A, Westermeyer J. The role of methadone maintenance in the management of opioid dependence and the prevention of aids. An international review. Praeger. Nueva York, 1989.

(3) Yancovitz SR et al. A randomized trial of an interim methadone maintenance clinic. American Journal of Public Health 1991, 81: 1185-1191.

(4) Ball JC, Ross A. The effectiveness of Methadone Maintenance Treatment. Ed. Springer-Verlag. Nueva York, 1991.

(5) Caplehorn JR, Bell J, Kleimbaum DG, Gebski VJ. Methadone dose and heroin use during maintenance treatment. Addiction 1993, 88(1): 119-124.

(6) Bobes J, Cervera S. Psiquiatría: Calidad de vida y calidad de asistencia. Ed Menarini. Barcelona, 1996.

(7) Alonso J, Prieto L, Antó JM. La versión española del SF36 Health Survey (Cuestionario de Salud SF-36): un ins- trumento para la medida de los resultados clínicos. Med Clin (Barc) 1995, 104: 771-776.

(8) World Health Organization. Option for the use of methadone in the treatment of drug dependence. WHO Division of Mental Health. Ginebra, 1989.

(9) Casas M. Presente y futuro de los programas con agonistas opiáceos. Adicciones 1995, 7(4): 411-422.

(10) Parrino MW. State methadone treatment guidelines. USD Health and Human Services. Rockville, 1993.

(11) Organización Mundial de la Salud. Clasificación Internacional de las Enfermedades: Trastornos Mentales y del Comportamiento. Ed. Meditor . Madrid, 1992.

(12) Ministerio de Sanidad y Consumo. Real Decreto 75/1990 de Enero por el que se regulan los tratamientos con opiáceos de personas dependientes de los mismos. B.O.E. de 23 de enero de 1990, pp. 21012103.

(13) Kokkevi A, Hartgers C. EuropASI: European adaptation of a multidimensional assesment instrument for drug and alcohol dependence. European Addiction Research 1995, 1: 208-210.

(14) Guerra D. Addiction Severity Index (ASI): un índice de severidad de la adicción". En: Trastornos psiquicos en las toxicomanias. Casas M, coord. Ed. NeurocienciasCitran. Barcalona, 1992.

(15) Ware JE, Gandek B. The SF-36 Health Survey: Development and use in mental health research and the IOOLA Project. International Journal of Mental Health 1994, 23(2): 49-73.

(16) Lobo A et al. Detección de morbilidad psíquica en la práctica médica: el nuevo instrumento EADG. Ed Luzán. Madrid, 1989.

(17) Mayfield D, McLeod G, Hall P. The CAGE questionnaire validation of a new alcoholism screening instrument. American Journal of Psychiatry 1974, 131: 1121-1122..

(18) Ward J, Mattick R, Hall W. Key issues in methadone maintenance treatment. New South Wales University Press. Sydney, 1992.

(19) Rodríguez-Martos A. Reflexiones sobre los programas de mantenimiento con metadona. Adicciones 1994, 6(4): 353-372.

(20) Torrens M, Castillo C, Pérez-Solá V. Retention in a low threshold methadone maintenance program. Drug Alcohol Dependence 1996, 41: 55-59.

(21) Marina P. Adictos a Opiáceos en Asturias: Un Estudio de seguimiento. Servicio de Publicaciones del Principado de Asturias. Oviedo, 1993.

(22) Kosten TR, Rounsaville BJ, Kleber HD. Multidimensionality and prediction of treatment outcome in opioid addicts: 2,5-year follow-up. Comprehensive Psychiatry 1987, 28: 3-13.

(23) D'Aunno T, Vaughm C. Variations in methadone maintenance treatment practices. JAMA 1992, 267: 253-258.

(24) Deglon JJ. Le traitment a long terme des héroinomanes parla metadone. WHO Ginebra, 1982.

(25) Giddey C, Dazord A, Gerin P. Etude preliminaire d'un programme de methadone dans un service public pour toxicomanes. Medicine et Higiene 1995, 53: 1521-1523. 
(26) Ryan CF, White JM. Health status at entry methadone maintenance treatment using the SF-36 Health Survey Questionnaire. Addiction 1996, 91, 1: 39-45.

(27) Torrens M, San L, Martínez A, Castillo C, Domingo A, Alonso J. Use of the Nottingham Health Profile for measuring health status of patients in methadone maintenance treatment. Addiction 1997, 92(5): 707-716.

(28) Ruiz de Apodaca J, Martínez H, Gutiérrez M, Perez J, Landábaso M. Estudio preliminar de la calidad de vida en adictos a la heroína en el programa de objetivos intermedios. XXI Jornadas Nacionales Socidrogalcohol. Bilbao, 1994.

(29) Bobes J, Gutiérrez M, Gibert J, González MP, Herraiz L, Fernández A. Quality of life in schizophrenia: long-term follow-up in 362 chronic Spanish schizophrenic outpatients undergoing risperidone maintenance treatment. European Psychiatry 1998, 13: 158-163.

(30) Ureña A, Rebollo P, Ortega F, Alvarez-Ude F, AlvarezGrande J. Health-related quality of life evolution in patients with functioning renal graft. Quality Life Research, 1997, 6: 733.

(31) Rebollo P, Ortega F, Ureña A, Bobes J, González MP, Sáiz PA. Quality of life in kidney-transplanted patients. Quality Life Research, 1997, 6: 707.

(32) Fresno A, González MP, Ferrer J, Bobes J. Calidad de vida y climaterio. IV Congreso Nacional de la Asociación Española para el Estudio de la Menopausia. Santander, 1996. 